\title{
Structural Network Analysis of Brain Development in Young Preterm Neonates
}

\author{
Colin J Brown ${ }^{\mathrm{a}, *}$, Steven P Miller ${ }^{\mathrm{b}}$, Brian G Booth ${ }^{\mathrm{a}}$, Shawn Andrews ${ }^{\mathrm{a}}$, Vann Chau ${ }^{\mathrm{b}}$, Kenneth J Poskitt ${ }^{\mathrm{c}}$, Ghassan \\ Hamarneh $^{\mathrm{a}}$
}

${ }^{a}$ Medical Image Analysis Lab, Simon Fraser University, Burnaby, BC, Canada

${ }^{b}$ Department of Paediatrics, The Hospital for Sick Children and the University of Toronto, Toronto, ON, Canada

${ }^{c} B C$ Children's Hospital, Vancouver, BC, Canada

\begin{abstract}
Preterm infants develop differently than those born at term and are at higher risk of brain pathology. Thus, an understanding of their development is of particular importance. Diffusion tensor imaging (DTI) of preterm infants offers a window into brain development at a very early age, an age at which that development is not yet fully understood. Recent works have used DTI to analyze structural connectome of the brain scans using network analysis. These studies have shown that, even from infancy, the brain exhibits small-world properties. Here we examine a cohort of 47 normal preterm neonates (i.e., without brain injury and with normal neurodevelopment at 18 months of age) scanned between 27 and 45 weeks post-menstrual age to further the understanding of how the structural connectome develops. We use fullbrain tractography to find white matter tracts between the 90 cortical and sub-cortical regions defined in the University of North Carolina Chapel Hill neonatal atlas. We then analyze the resulting connectomes and explore the differences between weighting edges by tract count versus fractional anisotropy. We observe that the brain networks in preterm infants, much like infants born at term, show high efficiency and clustering measures across a range of network scales. Further, the development of many individual region-pair connections, particularly in the frontal and occipital lobes, is significantly correlated with age. Finally, we observe that the preterm infant connectome remains highly efficient yet becomes more clustered across this age range, leading to a significant increase in its small-world structure.
\end{abstract}

Keywords:

Preterm, Brain Development, Structural Connectome, Network Measures, Neonates, Tractography

\section{Introduction}

The early configuration and development of the brain's structural network is not yet well understood. In vivo analysis of white matter connections typically requires a diffusion magnetic resonance (dMR) image of the brain which, for in utero subjects, presents significant challenges [28]. Preterm neonatal subjects provide an opportunity to study the early connectome without the difficulties associated with in utero imaging. Understanding the connectomes of these infants is doubly important due to the risk factors associated with preterm birth, including white matter injury and abnormal neurodevelopment [17]. Here, we examine a normative cohort of preterm neonatal infants scanned between 27 and 45 weeks post-menstrual age (PMA) and identify consistent topological and developmental trends in their structural brain networks. Our goal is to develop a better understanding of early brain configuration and growth which will enable future studies to better characterize abnormal development and injury.

${ }^{*}$ Corresponding author at: 9971 Applied Sciences Building (ASB), School of Computing Science, Simon Fraser University, 8888 University Drive, Burnaby, BC, Canada, V5A 1S6. Tel: 1-778-7824277, Email address: cjbrown@sfu.ca (Colin Brown)
Previous works have examined white matter development in young infants. Many early studies focused on voxel-wise measures of fractional anisotropy (FA) and mean diffusivity (MD) [10, 24, 35]. These works discussed the effects of myelination and reduction in brain water over time on increasing FA and decreasing $\mathrm{MD}[17,21]$.

Many other studies have looked at functional network development in young infants [19, 20, 22, 56]. Fransson et al., in particular, examined the resting-state functional network architecture of very young preterm infants (25 weeks mean gestational age) and found that only half of the number of resting-state sub-networks found in healthy adults were present at the preterm stage [20]. Recently, van der Heuvel et al. found that functional networks in preterm infants agreed well with the underlying anatomical structure [54]. In general, the relationship between functional networks and structural networks is complex and still not fully understood and there is still much work being done trying to explain causal relationships between the two [9, 49].

Some other recent works have focused on the examination of the structural connectome of young infants by performing tractography between numerous anatomical regions in the brain $[3,41,50,52,53,58,54]$. Takahashi 
et al. examined results of full-brain tractography qualitatively and described trends across postmortem infants between 17 and 40 weeks [50]. In order to quantify and organize tractography results, many studies abstract the connections in the brain as a network, where nodes typically represent anatomical regions and edges represent some measure of connectivity between those regions. Ball et al. examined connections in the thalamocortical network of preterm infants and showed that early birth correlated with reduced connectivity [3]. Pandit et al. studied the change in connection strengths across scan age and birth age on a cohort of preterm infants scanned as early as 47 weeks post-conception [41]. They reported that the frontal lobe showed a higher rate of development than other regions across their age group. They further noted that babies born prematurely showed lower overall cortical and sub-cortical connectivity than infants born at term.

Other preterm infant studies have looked into summarizing structural connectomes using network measures. Yap et al. examined the development of connectomes in young children, across a range of ages between 2 weeks and 2 years, using measures of network integration and segregation [58]. Tymofiyeva et al. used an atlas-free approach to analyze connectome development in preterm infants, children and adults, also employing network measures to capture topological changes $[52,53]$. Very recently, Ball et al. studied a specific network measure known as rich-club organization in a cohort of preterm infants [2]. They found that this rich-club structure, known to be present in adult brain networks, emerges as early as 30 weeks PMA.

Such network measures allow high-level summaries of brain network topology which have been shown to be useful, reliable bio-markers in discriminating normal and abnormal brain networks [32, 40, 47]. Rubinov and Sporns recently presented a comprehensive summary of such measures in relation to their use on structural and functional brain networks [44].

To date, network analysis of the entire preterm infant connectome, particularly over anatomically defined regions, has not been done for gestational ages earlier than term equivalent age. This gap is likely because of the difficulties in acquiring a large dataset of subjects at such a young age and because, until recently, brain atlases of young infants were not available. It is possible to perform a similar analysis without an atlas, as demonstrated by Tymofiyeva et al., however, this strategy makes it difficult to identify the anatomical significance of specific connections and sub-networks.

In this work, we compute structural brain networks for a cohort of young preterm neonates and analyze both local and global longitudinal trends. In performing this analysis, we observe that the brain networks of preterm infants show high efficiency and clustering measures across a range of network scales, a result seen in analogous studies of term infants at slightly older ages. We also note that the development of individual region-pair connections is often significantly correlated with age. In particular,
Table 1: Ages and counts for subjects and subject scans. Postmenstrual ages at birth are shown for the subjects while ages at time of scan are displayed for the scans.

\begin{tabular}{|c||c|c|c||c|c|c|c|}
\hline \multicolumn{1}{|c||}{} & \multicolumn{3}{c||}{ Counts } & \multicolumn{3}{c|}{ Post-Menst. Age (wks) } \\
\hline & Total & M & F & Mean & SD & Min & Max \\
\hline Subjects & 47 & 28 & 19 & 28.19 & 2.12 & 24 & 32 \\
Scans & 70 & 40 & 30 & 35.8 & 5.29 & 27 & 45 \\
\hline
\end{tabular}

we find that connections in the frontal and occipital lobes show high rates of development during this period. Finally, using established brain network measures [44], we see that the preterm infant connectome remains highly efficient and becomes more clustered across this age range, leading to a significant increase in small-worldness. As far as we are aware, this is the first connectome analysis of subjects as young as 27 weeks PMA and the first work to look at whole-brain network integration and segregation in a large, normative cohort of preterm infants.

\section{Materials and Method}

\subsection{Study Population}

To establish normative development of preterm structural brain connectivity, we selected "normal" infants from a prospective cohort described in Chau et al. [15]. This cohort consists of premature newborns born between 24 to 32 weeks post-menstrual age at the Childrens \& Womens Health Centre of British Columbia. Exclusion criteria included 1) congenital malformation or syndrome; 2) antenatal infection; or 3) large parenchymal hemorrhagic infarction $(>2 \mathrm{~cm})$ on head ultrasound scanning. This prospective study was approved by the University of British Columbia Clinical Research Ethics Board. The newborns enrolled in this cohort were evaluated with MRI scans in the neonatal period (outlined below) and had neurodevelopmental assessments at 18 months of age (corrected for prematurity) with the Bayley Scales of Infant and Toddler Development, Third Edition (BSID-III) [6] and the Peabody Developmental Motor Scales, Second Edition (PDMS-II) [18]. The 3 composite scores (cognitive, language and motor scores) of the BSID-III have a mean of 100 and a standard deviation of 15. The PDMSII provides a more sensitive assessment of motor function yielding gross, fine and total motor scores with a mean of 100 and standard deviation of 15 . To ensure a normative sample of preterm neonates, we included those infants without acquired brain injury on MRI (no white matter injury, no intraventricular hemorrhage) and with scores on all six composite measures of neurodevelopment within 1 standard deviation of the normal mean $(>85)$. After removing subjects with low cognitive test scores, detectable brain injury and low image quality (described below), the final number of subjects used in this study was 47 (28 males, 19 females). 


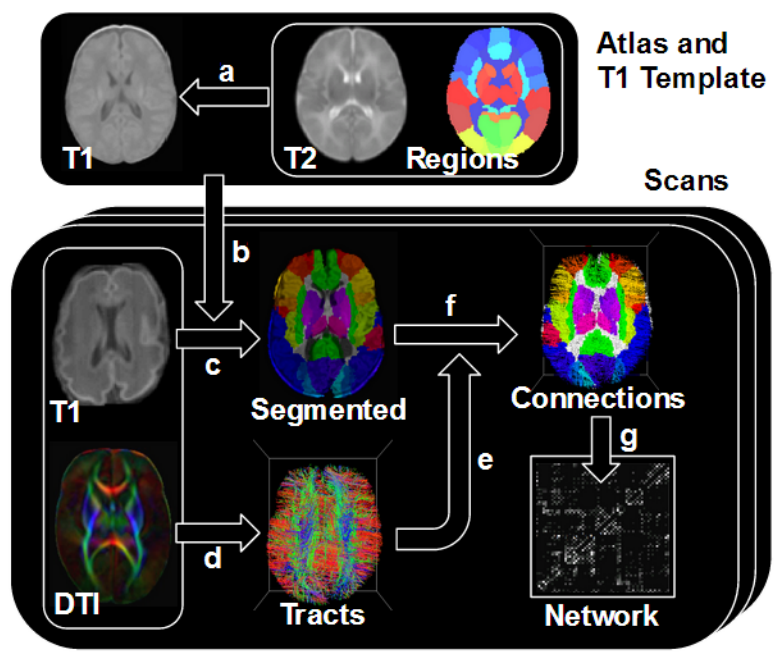

Figure 1: High level schematic representation of connectome pipeline. Arrows represent a) T2-T1 registration, b) T1-T1 registration, c) atlas-based segmentation, d) tractography, e) T1-tract alignment, f) assignment of tracts to region pairs, and e) tract counting or mean FA calculation

\subsection{Magnetic Resonance Imaging}

Each of the 47 preterm neonates were scanned within the first weeks of life once they were clinically stable. Twenty-three of these 47 infants were scanned again at term-equivalent age, with 2 to $15(9.49 \pm 3.45)$ weeks between scans. The resulting 70 structural and diffusion MRI scans cover the age range of 27 to 45 (35.8 \pm 5.29$)$ weeks PMA (Table 1).

Our MRI studies were carried out on a Siemens (Berlin, Germany) 1.5T Avanto using VB 13A software and included the following sequences: $3 \mathrm{D}$ coronal volumetric $T_{1^{-}}$ weighted images (repetition time [TR], $36 \mathrm{~ms}$; echo time [TE], $9.2 \mathrm{~ms}$; field of view [FOV], $200 \mathrm{~mm}$; slice thickness, $1 \mathrm{~mm}$, no gap) and a 3D axial volumetric diffusion tensor image set (TR $4900 \mathrm{~ms}$; TE $104 \mathrm{~ms}$; FOV $160 \mathrm{~mm}$; slice thickness, $3 \mathrm{~mm}$; no gap) with 3 averages of 12 noncolinear gradient directions over 2 diffusion weightings of 600 and $700 \mathrm{~s} / \mathrm{mm}^{2}$ (b-value), resulting in an in-plane resolution of $0.625 \mathrm{~mm}$. Each diffusion weighted image set was preprocessed using the FSL Diffusion Toolbox (FDT) pipeline $^{1}$ and tensors were fit using RESTORE [14].

An experienced neuroradiologist (K.P.) reviewed the resulting MR images for presence of white matter injury (WMI), intraventricular hemorrhages (IVH), and poor image quality due to subject motion. The presence of WMI was identified using a system found to be predictive of adverse neurodevelopmental outcome at 12 to 18 months of age [34]. We noted IVH using the protocol of Papile et al. [42]. The 70 scans used in this study were selected so as to be of sufficient quality and be free of these pathologies.

${ }^{1}$ http://fsl.fmrib.ox.ac.uk/fsl/fslwiki/FDT

\subsection{Atlas Based Segmentation}

We used an atlas-based method to segment each scan. A brain region atlas of $\mathrm{T} 2 \mathrm{MR}$ images from the IDEA group at University of North Carolina (UNC) School of Medicine, Chapel Hill was aligned to each skull-stripped T1 image in the dataset [46]. The T2 atlas was used due to the lack of availability of an anatomically labelled T1 brain region atlas of young infants at the time of this study. The T2 UNC atlas was constructed from 95 subjects, born between 33 and 42 weeks, with scans taken between 38 and 46 weeks PMA (41.5 \pm 1.7 weeks). The atlas contains 90 (45 per hemisphere) anatomically defined cortical and sub-cortical gray matter regions (Table A1), each with an average volume of $4.7 \pm 3.1 \mathrm{~cm}^{3}$.

We first register the UNC neonatal T2 atlas to a T1 template of infant brains, followed by aligning the $\mathrm{T} 1$ template to each subject's T1 scans. We perform the registration this way as inter-modality registration generally presents more challenges than intra-modal registration and has a higher risk of image registration error. Aligning the T2 atlas to a single $\mathrm{T} 1$ template allows the visual examination of a single result to ensure that the inter-modality registration was accurate. Furthermore, aligning the T2 atlas directly to each $\mathrm{T} 1$ subject would have been very time consuming due to the extra computation required for inter-modality registration. We register to a $\mathrm{T} 1$ template that is a $3 \mathrm{D}$ time-slice selected from the 4D Imperial College London (ICL) infant atlas, an atlas constructed from 204 premature neonates between 28 and 44 weeks [45]. We registered to the $3 \mathrm{D}$ time-slice corresponding to 38 weeks PMA, the youngest age of the UNC dataset.

Each registration was performed first by an affine transformation using FMRIB's Linear Image Registration Tool (FLIRT) [27], then a deformable transformation using the MATLAB Image Registration Toolbox (MIRT) ${ }^{2}$. For the inter-model registration, both registration steps used a mutual information similarity metric since it is known to be a good choice for such registration problems [43]. For the intra-modal registrations, a cross-correlation similarity metric was sufficient. Anatomical regions in the UNC atlas were propagated to the 38 -week T1 template and then to each $\mathrm{T} 1$ image in the dataset by applying the same transforms obtained from the registration steps.

Prior to registration, each infant's T1 image was neckstripped using a manually selected slice-plane, then skull stripped first using BET [48] and then using an agematched, rigidly aligned brain mask from the ICL 4D T1 atlas. The combination of this skull stripping and registration gives us an anatomical segmentation of each T1 image in it's native frame of reference which, upon careful visual inspection, was found to be highly accurate.

\subsection{Connectome Mapping}

We perform whole-brain tractography on each infant's DTI using TrackVis [55]. We seed streamlines at all voxels

\footnotetext{
${ }^{2}$ https://sites.google.com/site/myronenko/research/mirt
} 
Table 2: Network measures used in this study.

\begin{tabular}{|c|c|c|}
\hline Category & Network Measure Name & Acronym \\
\hline \hline Connectivity & Mean Weighted Nodal Degree & MWD \\
\hline Integration & Characteristic Path Length & CPL \\
& Global Efficiency & GE \\
& Local Efficiency & LE \\
\hline Segregation & Clustering Coefficient & CC \\
& Modularity & ML \\
\hline Small-World & Small-Worldness & SW \\
\hline
\end{tabular}

with FA greater than 0.1 since this is the value of the noise floor [29]. By choosing this FA threshold, we obtain all possible tracts that can be extracted from the DTI. However, this strategy leads to a developmental bias as the number of streamline seeds depend on the brain volume and overall FA levels in the DTI. We will address this bias later in section 2.6. We spline filter all tracts and then align them to the infant's T1 image space.

The number of streamlines with end-points in each pair of anatomical brain regions were counted to create an $n$ by $n$ connectivity matrix, $C^{k},(n=90)$ for each scan, $k$, where $c_{i j}^{k}$ is the number of tracts in scan $k$ between regions $i$ and $j$. Note that each streamline is counted only once as we count a streamline based its end points and not whether a streamline goes through a region. Also, streamlines connecting a region to itself are ignored, causing the diagonal entries of the matrix to be 0 . While the streamlines provided by TrackVis are presented with an implied direction, the direction of diffusion measured by the underlying DTI is ambiguous. Thus, we discard the directional information in each network by summing each connectivity matrix with it's transpose, imposing symmetry.

Using this symmetric connectivity matrix, we define our structural connectome as a network $G^{k}=\left(V^{k}, E^{k}\right.$ ) (for each scan $k=1 \ldots 70)$. In this network, the brain regions are represented as a set of nodes $V^{k}=\left\{v_{1}^{k}, \ldots, v_{n}^{k}\right\}$. The connections between brain regions are represented by edges $E^{k}=V^{k} \times V^{k}=\left\{\left(v_{1}^{k}, v_{2}^{k}\right), \ldots\right\}$ where $e_{i j}^{k}=\left(v_{i}^{k}, v_{j}^{k}\right)$ represents the connection between regions $i$ and $j$. The connectome network is then weighted by assigning a weight $w\left(e_{i j}^{k}\right)$ to each edge $e_{i j}^{k}$ corresponding to the amount of structural connectivity between the two regions. This connectome weighting usually takes one of two forms: we can assign an edge weight based on the raw number of streamlines between regions (i.e., $w\left(e_{i j}^{k}\right)=c_{i j}^{k}$ ), or we can examine tract "integrity" by using the mean FA computed over the $c_{i j}^{k}$ streamlines as an edge weight [3]. We will examine both weighting schemes and refer to their networks as tract-count and mean-FA connectomes respectively.

Figure 1 summarizes, at a high level, the pipeline used to generate each brain network.

\subsection{Network Analysis}

The structural connectomes defined in the previous section are networks and it has become popular to examine these networks using various network measures $[2,32$,
40, 44, 47, 58]. The collection of network measures used here are given in Table 2. At a high level, these measures capture four intrinsic properties of networks: connectivity, segregation, integration, and small-worldness. Below, we describe the relationship between these measures and the network properties they capture. For a more extensive discussion of network measures, we refer the reader to [44].

To begin, the network property of connectivity refers to the number of connections between nodes. A highlyconnected network has more edges, or more highlyweighted edges, than a marginally-connected network. In the context of our connectomes, connectivity directly relates to the number of streamlines between brain regions, or the average FA value of tracts between two regions. We can capture this network connectivity using the mean weighted nodal degree (MWD) measure, which reports the average number of connections for a network node. Computing MWD involves computing each node's degree: the sum of edge weights for edges connected to a node. The MWD is then average of these node degree values across the network.

Complementing network connectivity is the property of network integration. While connectivity refers to the amount of connectivity in a network, integration refers to how densely connected all nodes are to one another. For example, a fully connected network has high network integration as each node is connected to every other node. On the other hand, a network with fully disconnected parts has low network integration. In the context of our connectomes, network integration relates to how interconnected all brain regions are to one another. The most common network integration measures are characteristic path length (CPL), global efficiency (GE), and local efficiency (LE). A network's CPL relates to the length of the shortest paths through the network, where path length is the inverse sum of the edge weights for edges along a path. The CPL measure is computed as the average of these shortest path lengths between all pairs of nodes in the network [57]. Similarly, GE measures network integration using shortest paths and, in fact, is simply the average inverse of the shortest path lengths [44]. Both of these measures examine shortest paths through the entire network. However, we can also look at shortest paths locally by measuring the length of the shortest path between the neighbours of a given node. These local shortest path lengths are used to compute LE. Like GE, LE is the average inverse of the shortest path lengths, but LE uses these local shortest paths instead of the overall shortest paths [31].

Conversely to network integration, network segregation refers to how much a network is organized into a collection of sub-networks. For example, a fully connected network, where each node is equally connected to all other nodes, has low network segregation; whereas a network with fully disconnected parts has high network segregation. In the context of our connectomes, network segregation relates to what degree a brain's anatomical regions are arranged into small, distinct clusters. There are two popular measures 
that capture network segregation: modularity (ML), and clustering coefficient (CC). The ML measure captures network segregation by measuring the number of connections (i.e. the sum of the edge weights) within a sub-network and comparing that to the number of connections exiting the same sub-network. ML is then taken as the maximum of these ratios across all possible sub-networks [36]. As it is infeasible to create all possible sub-networks, ML is typically approximated using an algorithm like that proposed by Newman [37]. Similarly, the CC of a network captures its segregation, but unlike ML, CC can be computed exactly. A network's CC captures the fraction of a node's neighbours that are also connected to each other. These fractions at each node are then averaged over the network to give the overall network CC. Each node's CC can also be weighted by the product of the relevant edge weights to obtain a weighted version of this measure [57, 39]. This weighted CC is what is calculated in our study.

Finally, the small world property of a network builds off of these simpler network properties to capture something more complex. A small work network is one that has evidence of both segregation and integration, where nodes are grouped into sub-networks but those sub-networks are also connected in an organized way [4]. A small-world network is known for its efficiency; it keeps high connectivity between nodes with a minimal number of connections. This small world property can be captured using the smallworldness (SW) measure, which is simply a normalized ratio between $\mathrm{CC}$ and $\mathrm{CPL}$ (where the normalization is discussed in Section 2.6). Many complex networks, including functional and structural brain networks of animals, have been shown to have this small-world property. It has also been shown that SW is reduced in brain networks of patients with neurodegenerative diseases, suggesting that SW might be an integral property of a well functioning brain [4]. It is not yet known when SW emerges in human brain networks and, given the early ages of the subjects in this study, we have the opportunity here to study how SW develops.

\subsection{Normalization}

By seeding tractography at every voxel with $\mathrm{FA}>0.1$, the number of tracts generated differs between scans. In particular, brain volume and white matter maturation may affect the number of tractography seeds and thus the edge weights in our connectomes. Since the focus of this work is more on the structural topology of white matter than on its volume or degree of maturation, we normalize each connectome in a way that minimizes the effect of these variables. Two different normalization strategies are adopted here and used where most appropriate.

When analyzing changes in individual connections (section 3.2) with respect to age, we normalize each connectome by dividing by the total number of tracts. Edge weights in each normalized connectome then represent relative connectivity and are independent of seeds used when performing tractography.
We use a different strategy when analyzing results from network measures (sections 3.3, 3.4). While normalizing the edge weights in the connectome removes the tract count bias, an additional bias factor exists due to the fact that a random, 'null-hypothesis' network may have a nonzero value for a given network measure. We address this additional bias in the network measures by comparing each measure's value to one computed from a similar network where edges have been placed randomly. These randomlybuilt networks are generated with the same number of nodes as our connectome and the same distribution of node degrees, thereby removing any bias due to connectome magnitude as well as the fraction of a measure's value that arises by chance [44]. In this work, we compute ten random networks for each connectome and the network measures of these random networks are averaged. The normalized network measures are then obtained by dividing the original, unnormalized, measures by the average obtained from the randomly-built networks.

Note that we do not normalize the mean-FA connectomes because the FA values for a region-pair connection are averaged across all its $c_{i j}^{k}$ streamlines, making it invariant to the number of tractography seeds used. Also, we do not wish to normalize for increasing FA since examining change in FA and comparing it to change in tract count is the exact reason these connectomes were computed.

\section{Results}

With a brain network computed for each subject scan, we performed four experiments designed to expose important aspects of early connectome development in preterm infants. We first investigate group-wise properties of the preterm connectomes using high-level network measures and compare the results to network measure values from a group of infants born at term (section 3.1). We then examine longitudinal trends in for each region-pair connection using a linear mixed-effects model (section 3.2). Finally, we look at network measures in the preterm infant brain networks across time, first as trends within the entire set of scans (section 3.3), and then as changes between scans in each subject (section 3.4).

\subsection{Groupwise Connectome Analysis}

Our first goal is to show that our results are reasonably consistent with results from a similar study. In particular, we repeat an experiment by Yap et al. originally done on a group of young infants born at term [58]. By doing so, we are able to both validate our connectome pipeline against another from a different group and compare preterm infants to those born normally at term with similar scan ages. Such a validation is important as it has been suggested that comparing different connectome studies is difficult due to the large number of methodological choices and parameters [5]. 

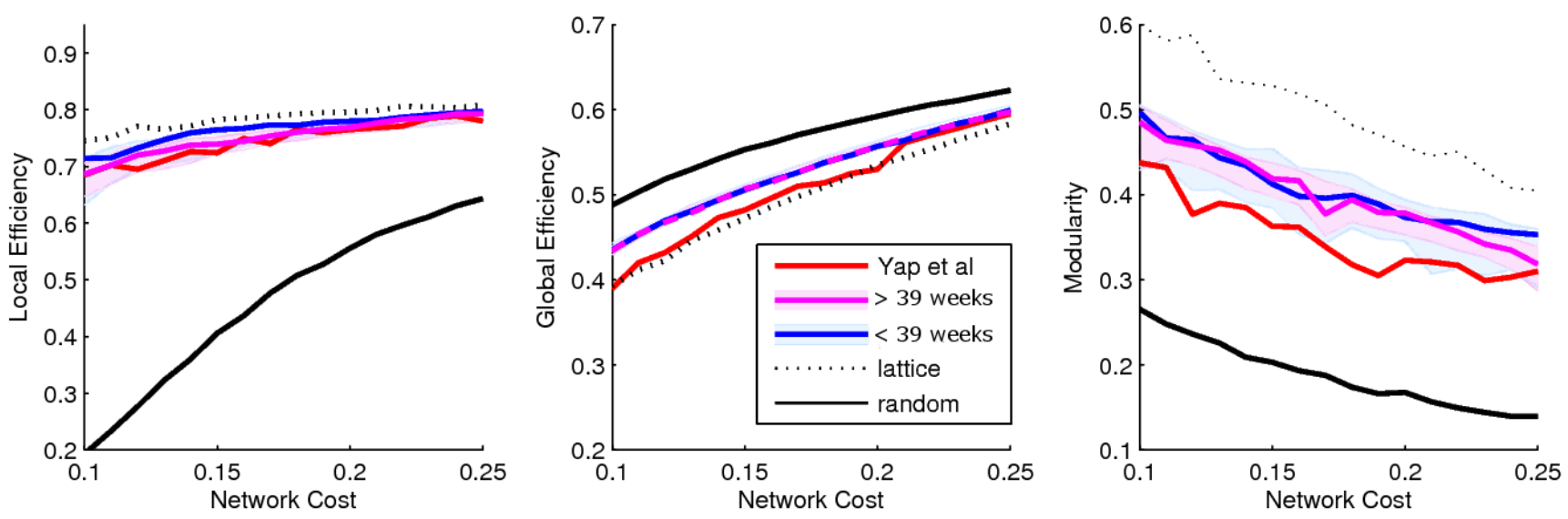

Figure 2: Backbone network cost v.s. local efficiency, global efficiency and modularity. Network cost is varied by applying different thresholds to the group connectivity SNR matrix (see text). Network measures are plotted for three groups of infant scans. Results from Yap et al. of a group scanned 2 weeks after normal term birth (red) are plotted next to group of preterm infants scanned around term-equivalent age (magenta). A third group of preterms scanned before 39 weeks PMA (blue) is also shown. Bootstrap-estimated confidence intervals for our preterm groups appear in light magenta and light blue. Note that the preterm and term groups show a similar trend in connectome structure with some slight differences in global efficiency and modularity. We elaborate on these differences in section 4.

In attempt to make the comparison fair, a subset of scans from our dataset is selected to match the distribution of ages from the term group in [58] as closely as possible. By selecting scans acquired after 39 weeks PMA, we obtain a group of scans with mean age $41.7 \pm 1.9$ weeks; very close to the mean age of the term group at $41.6 \pm 1.7$ weeks. We also compare these groups to a third group, comprised of all remaining preterm scans, taken before 39 weeks (32.6 \pm 3.1 weeks).

The connectome results from Yap et al. rely on the concept of a backbone network computed from a group of subjects $[23,58]$. This backbone network captures connections that are consistent across the group through the use of a signal-to-noise (SNR) connection matrix. For each group, $\mathcal{G}$, an element in the SNR matrix is defined as,

$$
S N R_{i j}^{\mathcal{G}}\left(c_{i j}^{k}\right)=\frac{M E A N_{k \in \mathcal{G}}\left(c_{i j}^{k}\right)}{S D_{k \in \mathcal{G}}\left(c_{i j}^{k}\right)} .
$$

where $S D$ is the standard deviation across subjects. Note that to stay consistent with Yap et al., we only use the tract-count connectomes when computing this matrix. The backbone network is then built from the SNR connection matrix by thresholding over a range of values to produce binary matrices of varying network costs (i.e., the fraction of possible edges present after thresholding). Each binary matrix can be interpreted as a network in the fashion described earlier and we can compute the GE, LE and ML for each of them. These three measures of the thresholded backbone network are plotted versus network cost in Figure 2. Also plotted are the GE, LE, and ML for the special cases of random networks (where edges are distributed randomly) and lattices (where nodes are connected to form a network with a grid-like structure). We also extended the analysis of Yap et al. by using statistical bootstrapping to estimate confidence intervals for all three network measures (using 50 bootstrap iterations of
39 samples with replacement). These confidence intervals give us further insight into the stability of the backbone network structure.

As we would expect, both LE and GE rise with greater network costs (i.e. more edges) in all groups. Also expected is that the three measures for our connectomes fall in the range between the extreme cases of random networks and lattices. These outcomes are consistent with those reported by Yap et al. for infants born at term and unsurprising since we expect the brain to have a strong local structure combined with cross-network connections. However, we do see notable differences between our preterm connectomes and those term infant connectomes analyzed by Yap et al.. First, the GE of our two preterm groups is higher for most network costs than seen in the group born at term. Second, the ML of our preterm infants is significantly higher than those reported for term infants. Possible interpretations of these differences are discussed later in section 4 .

\subsection{Per-Connection Analysis}

In order to understand inter-region white matter development over the range of scan ages in our group, we examined each connection in our tract-count and mean-FA connectomes across time. Since certain subjects were scanned twice, we required a statistical model that assumes possible intra-subject biases. Here we employed a linear mixedeffects model, a generalization of a linear regression model that assumes possible correlation between scans from the same subject [38]. A model of this kind is fit to the tract counts and mean FA values of each region-pair connection across PMA.

Our particular interest is to discover which region-pairs connections are consistently exhibiting longitudinal trends across the cohort. To test for this, we compute a confidence interval (CI) for our linear model's slope and check 



Tract Count
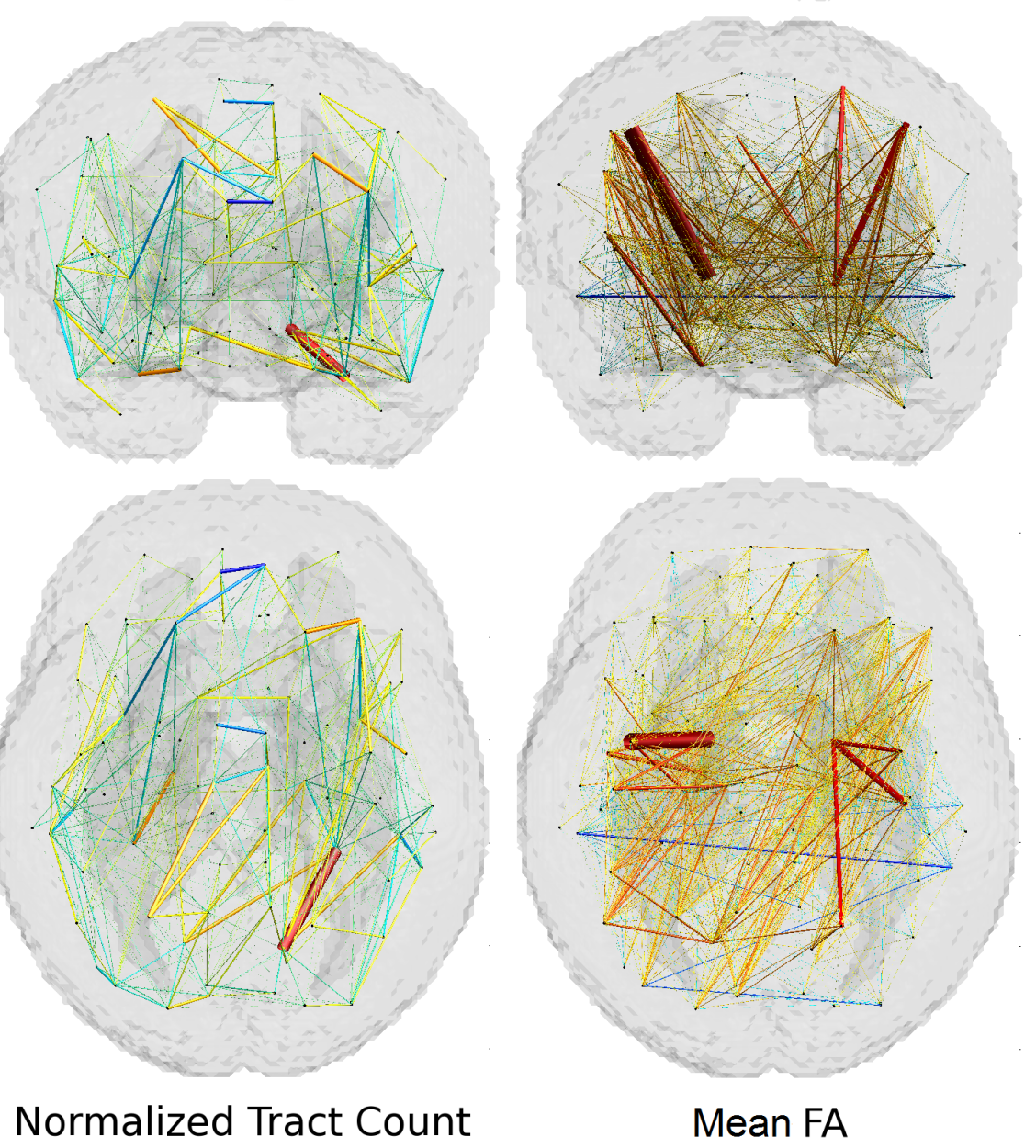

Mean FA

Figure 3: Edge weight slopes for linear mixed-effects models fit longitudinally to tract-count, normalized tract-count, and mean-FA connectomes. Connections are mapped spatially onto UNC atlas. Connections are only displayed if the $99 \%$ CI of their longitudinal slope did not include zero (after correction for multiple comparisons). Edge colour maps to the linear model's slope value and thicker lines map to greater slope magnitudes.

to see if that CI contains 0 . If the CI falls strictly above zero (or strictly below zero) then we can say it is significantly likely that the values are increasing (or decreasing) across time. To correct for multiple statistical computations across the $4050=90 \times 90 / 2$ region-pairs, we adopt the approach of Benjamini et al. to select significant CIs using a False-Discovery-Rate-based criterion [8].

After correction for multiple hypothesis testing, 664 (of a possible 4050) region pairs had tract counts with slopes significantly likely $(p<0.01$, FDR corrected [8]) of being non-zero. The locations of these region pairs and the magnitudes of their slopes are displayed spatially in Figure 3. 571 of these slopes were positive implying that a majority of these connections are gaining tracts over time.

After normalization of total number of tracts in each connectome, the same analysis was run. As the edge 


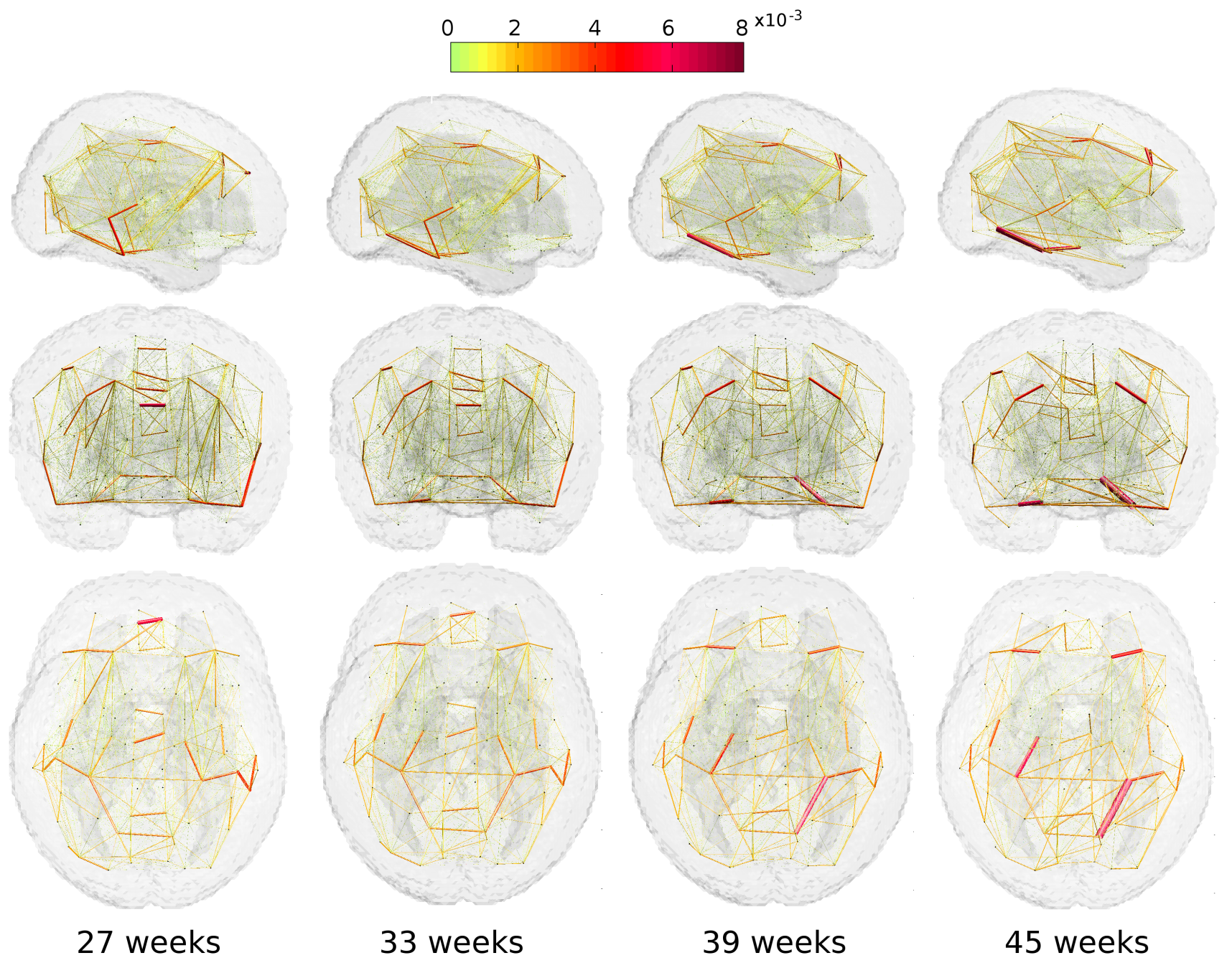

Figure 4: Predicted normalized tract-count connectomes exposing relative changes in network topology from 27 to 45 weeks PMA. Edge weights are predicted at four ages using linear mixed-effects models fit to each connection across scans. Connections with at least $0.1 \%$ of the brain's fiber tracts are rendered spatially over UNC atlas from sagittal, coronal and axial views (top to bottom). Each connection's thickness and colour represents the predicted fraction of tracts in the brain that connect each region pair.

weights in these normalized connectomes convey the relative strength of connections between regions, we expect a greater balance between those increasing in strength over time and those decreasing in strength. Of region-pairs with slopes found to be significantly likely of being non-zero, only 211 were positive where as 250 were negative. As expected, many of the region-pair connections with steeply increasing tract-counts also have steep positive slopes in the normalized tract count connectome (Figure 3). Also expected is that connections with negative slopes in the normalized connectomes are frequently between regions where there was no significant positive trend in the unnormalized connectomes.

Of the 211 connections with positive slopes in the normalized connectomes, $40 \%$ are within the left hemisphere, $38 \%$ are within the right hemisphere and $22 \%$ are interhemispheric. Connections with negative slopes are distributed between hemispheres in a similar fashion. We also find that 34 region pairs in the frontal lobe (atlas regions 1 through 30, Table A1), had normalized tract counts significantly increasing with age. Meanwhile, the occipital lobe (atlas regions 37 through 66 ), there are 28 such connections. The development of connections in these lobes was noticeably higher than the 23.0 \pm 5.73 developing connections obtained for a random selection of 30 regions (over 1000 random samplings with replacement).

We also examined the split between developing corticocortical connections and those connections with deep gray matter regions. In the normalized connectomes, $\sim 59 \%$ of edges with significantly increasing tract counts were between cortical regions versus only $\sim 5 \%$ of edges between deep gray matter regions (with the remainder of edges between the cortex and the deep gray matter). This comparison may be unfair, since 76 of 90 atlas regions are within the cerebral cortex. However, even when weighted by the model slopes, (i.e., the relative tract-count increases 
at each edge, ) these cortico-cortical connections represent $\sim 72 \%$ of the total rate of tract count increases in the infant brains.

The differences in brain volumes between different subjects in each scan makes it difficult to compare tract lengths between scans in a meaningful way. However, using the centroids of the UNC atlas regions we can determine the relative distances between region pairs in a common space. The UNC atlas is $115 \mathrm{~mm}$ long along the coronal axis with centroids an average of $48.6 \pm 16.2 \mathrm{~mm}$ apart. The two most distant regions are $98 \mathrm{~mm}$ apart. Using these Euclidean distances as an indicator of relative tract lengths between regions we can explore the development of spatially local versus spatially distant connections. Of edges with significantly increasing weights in the normalized tract-count connectomes, $\sim 50 \%$ were between region pairs less than $30 \mathrm{~mm}$ apart. However, some long-range connections were also found to be developing, with 12 significantly increasing region-pairs greater than $60 \mathrm{~mm}$ apart and 2 greater than $70 \mathrm{~mm}$ apart.

Finally, the same statistical analysis was carried out on the mean-FA connectomes. For these connectomes, 840 region-pairs showed significantly increasing FA whereas 169 showed significantly decreasing FA. While there are more region-pair connections with significantly increasing FA than those with increasing tract count, $75 \%$ of regionpairs with significantly increasing tract count also show significantly increasing FA. Furthermore, $82 \%$ of regionpair connections with relatively increasing tract counts (observed in the normalized connectomes) also show significantly increasing FA. (At random, we only expect only $21 \pm 1.6 \%$ and $21 \pm 2.8 \%$ of connections to overlap in these two cases, respectively.) Despite appearing to have very different configurations in Figure 3, the longitudinal trends in the tract count and FA connectomes are certainly related. We discuss possible reasons for discrepancies in section 4 .

To visualize the changes in brain network topology across time, we used our linear mixed-effect models for each connection to estimate tract counts at four ages spanning our cohort's age range. The resulting predicted brain networks are shown in Figure 4. These four evaluated brain networks show connections gaining and losing relative connection strength over time. Certain connections in particular show high rates of development, particularly around the lingual, fusiform, and parahippocampal gyri (see Figure A.1 for locations of these regions). Others, like the connection between the medial parts of the left and right superior frontal gyri show a rapid decrease in relative strength, implying that the number of tracts between these regions are not increasing as quickly as between a typical region-pair.

\subsection{Network Measures Versus Age}

In order to summarize how the organization of the structural connectome develops over time, we plot measures of structural connectivity, segregation, integration and smallworldness versus age in Figure 5. We used the Brain Connectivity Toolbox to compute all reported network measures [44].

For the unnormalized network measures, MWD was found to have a significant positive correlation with age, while CC and CPL are significantly correlated with age, positively and negatively, respectively. These results confirm the hypothesis that most region-pair connections are gaining tracts over time, likely due to brain volume increases and white matter maturation.

In the case of the normalized network measures, we found that normalized CPL showed no significant correlation with age and stays roughly constant. This implies that as the brain develops, the topological distance between any two regions is remaining constant on average. However, normalized CC is significantly positively correlated with age, implying that regions are becoming segregated into distinct clusters. The combination of these results suggest that over this age range, the preterm infant brain is organizing itself into clustered sub-networks while maintaining its larger scale, cross-network connections. The SW measure, having been computed by dividing normalized CC by normalized CPL, (which, we note, also makes it a normalized measure, ) shows a similar significant positive correlation. This implies that the structure of the infant connectome is becoming more small-world, independent of total number of tracts.

We summarize SW slope CIs across tract count, normalized tract count and mean-FA connectomes in Table 3 . Note that SW slope CIs for normalized and unnormalized tract count connectomes are very similar. This validates our assumption that the SW measure is already independent of overall edge-weight magnitude. Also note that the mean-FA connectomes lack a clear trend of increasing SW with age. We discuss potential reasons for the appearance of this result in Section 4.

\subsection{Intra-Subject Network Changes}

With 23 subjects scanned twice, we can examine intrasubject longitudinal trends of these structural connectomes. In Figure 6, we show the differences in the network measure values versus the time between scans.

For the unnormalized measures, all subjects displayed increasing MWD and CC while CPL decreased between scans. This result is what we would expect based on the inter-subject trends in the previous experiment. Further, changes in MWD and CC are significantly $(p<0.01)$ positively correlated with age, while changes in CPL are significantly negatively correlated with age. Thus, longer durations of time linearly relate to larger changes in the measures.

For the normalized measures, CPL values are scattered above and below zero. This result suggests that, compared to random networks, about as many subjects became relatively less efficient as those that became relatively more efficient. By applying a t-test, we found that 

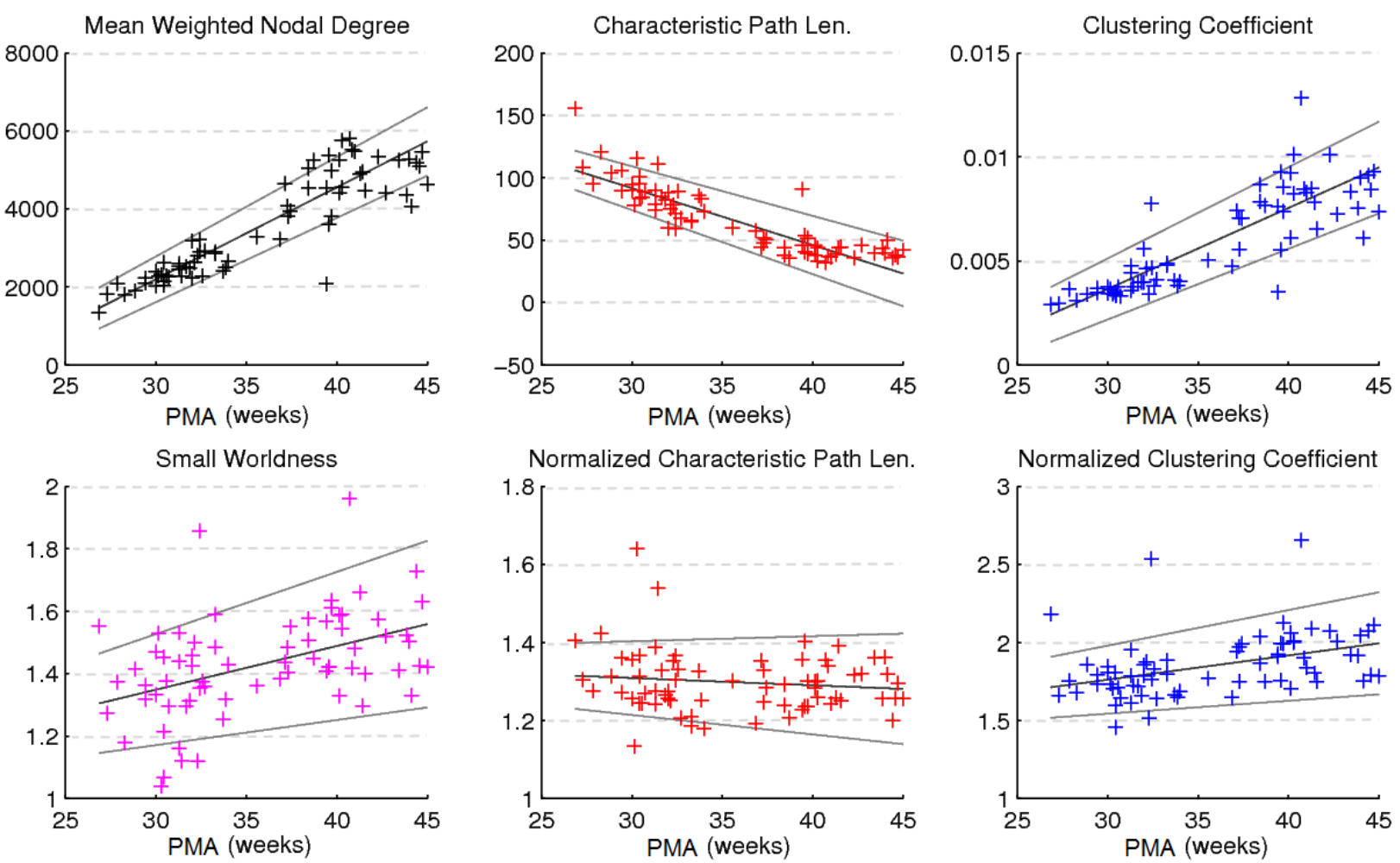

Figure 5: Mean degree and other network measures plotted against age at time of scan. Linear mixed-effects model fit to each measure (black) and are displayed with the $95 \%$ confidence interval for the parameters of each linear model (gray). Note the significant increases in normalized clustering coefficient and small-worldness across this age range.

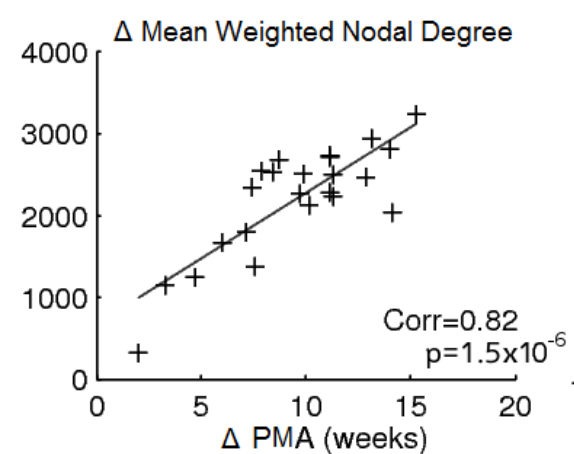

$\Delta$ Small Worldness

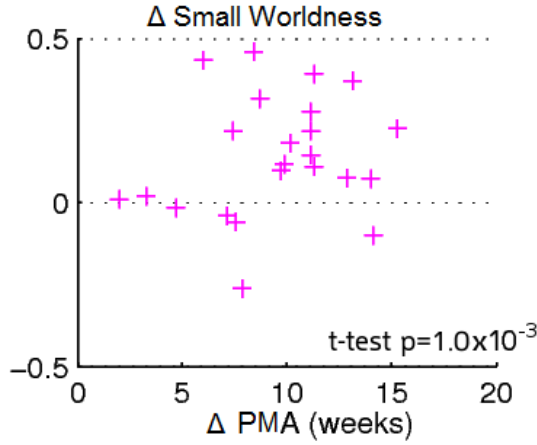

$\Delta$ Characteristic Path Len.

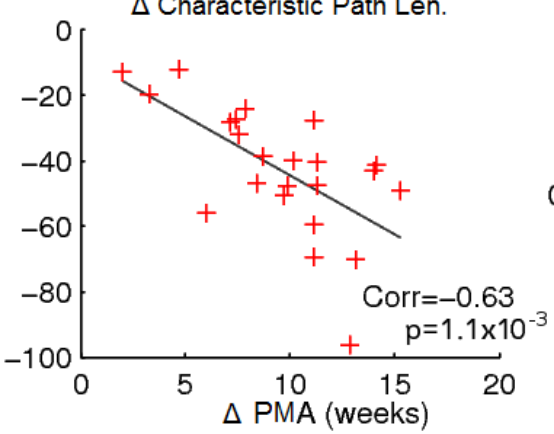

$\Delta$ Normalized Characteristic Path Len.

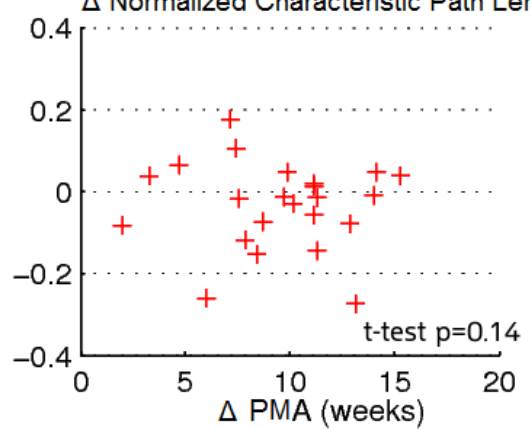

$\Delta$ Clustering Coefficient

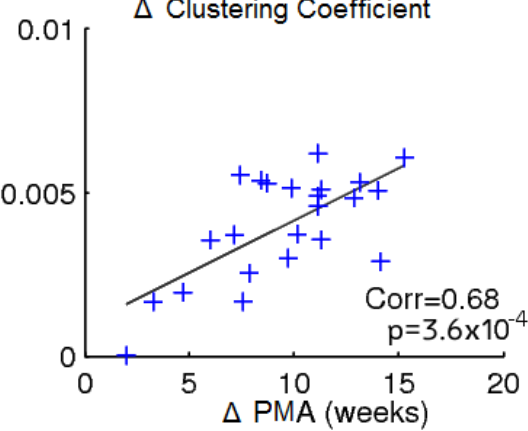

$\Delta$ Normalized Clustering Coefficient

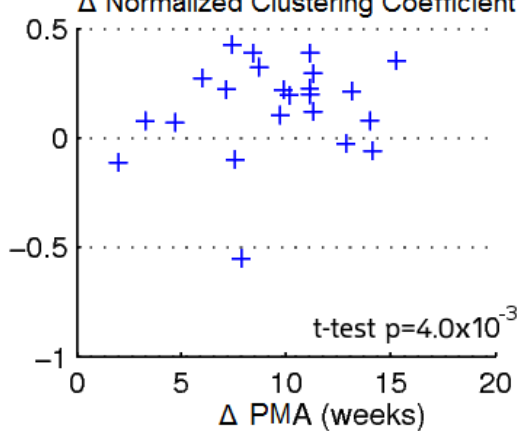

Figure 6: Change in network measures between an infant's first and second scan versus number of weeks between scans. Pearson's correlation reported for changes in unnormalized measures (top row). For each normalized measure (bottom row), a t-test is run to find if the mean connectome change between scans is significantly different from zero. p-values are reported for each measure. Note that normalized clustering coefficient and small-worldness show a significant increase between scans. 
Table 3: Summary of small-worldness (SW) trends in tract count, normalized tract-count and mean-FA connectomes. The middle three columns report slope estimates and 95\% CI bounds for linear mixedeffects model fit across scans. The right-most column reports t-test $\mathrm{p}$ values for the hypothesis that SW does not change over time for scans from the same subjects. Note that SW increases significantly for the tract-count connectomes but not the mean-FA connectome.

\begin{tabular}{|c||c|c|c||c|}
\hline & \multicolumn{3}{||}{ Mixed Model Slope CI } & Subject $\Delta$ \\
\hline Tract-Count & 0.0081 & 0.0140 & 0.0199 & 0.0017 \\
Norm. Count & 0.0070 & 0.0133 & 0.0196 & 0.0032 \\
Mean-FA & -0.0044 & -0.0007 & 0.0030 & 0.6498 \\
\hline
\end{tabular}

the mean normalized CPL value was not significantly different from zero $(p=0.1333)$. Normalized CC, however, increased between scans in most subjects which, in turn, caused SW to increase. Using t-tests, the mean values of both measures were found to be significantly greater than zero ( $p=0.0056$ and $p=0.0017$ respectively). Both this experiment and the previous one strongly suggest that SW increases with PMA in the tract-count connectomes of preterm infants (Table 3).

\section{Discussion}

In this work, we found that our dataset of preterm infants, represented as two SNR group-connectomes of younger and older scans, showed similar responses to thresholding as a group of similarly aged infants born at term and studied by Yap et al. [58] (section 3.1). The comparisons between the infant groups and random and lattice networks resulted in the predicted outcomes and all three groups of infant connectomes exhibited a balance of network integration and segregation. Despite these similarities, Yap et al. reported, at certain network costs, lower ML and GE in the term group than we find in the preterm group. These results seem to disagree with our later analysis on how network integration and segregation develop over time. However, it is likely the case that differences between connectome pipelines can explain this discrepancy.

One important difference between the two connectome pipelines is the way in which tract-count connectomes are constructed. In Yap et al.'s work, the weight of each tractcount connectome edge represented the number of tracts passing through a pair of atlas regions. In contrast, we only count a tract based on what regions its end-points are in. So, for the same scan, Yap et al.'s method will report strictly larger edge weights, especially for regions that are intermediaries on long-range fiber tracts. This bias towards intermediate regions means that small sections of these long-range connections will dominate the topology of the backbone network more so than their full, long-range connection.

This counting bias explains why, at low network costs, GE was higher in the age-matched preterm group compared to the group born at term. In the connectomes computed by Yap et al. , long-range connections, which increase GE, will be counted less than their corresponding sub-connections and thus will be less likely to be included in the backbone network. Further, we see that for higher network costs, these long-range connections are preserved in the backbone network and the difference in GE vanishes.

We also found that at most network costs, the ML computed by Yap et al. was lower than what we computed in the age-matched preterm group. We expect this is also due to the counting bias mentioned above. The edges that receive larger weights by Yap et al.'s method are likely to have low weights in our connectomes, since these intermediate connections are unlikely to have a large number of similar tracts that terminate in the two corresponding regions. These intermediate connections are also likely to connect regions along long-range fiber bundles, thereby reducing network segregation and ML.

Given that differences in connectome construction can account for discrepancies in network measures between group backbone networks in the two studies, the degree of network segregation and integration in infants born preterm versus those born at term may, in fact, be similar. It has been well-established that preterm birth is associated with reduced FA and increased mean diffusivity, suggesting reduced structural connectivity [1, 3, 24]. Our results may complement those findings by further showing that this reduced connectivity does not affect the overall organization of the connectome, or that the differences in organization are subtle enough that they cannot be discerned from measures of the backbone network. The second of these two explanations seems more likely as we did not see a difference in ML between our younger and older preterm groups despite seeing a trend of increasing segregation with time in section 3.3 .

An examination of individual region-pair connections (section 3.2) revealed a number of connections exhibiting relatively larger increases in tract count with age. If we assumed the rate of tract development was balanced, we would expect to see a 50-50 balance between the number of tracts gaining and losing importance in the normalized connectomes. Instead, we observed that $\sim 46 \%$ of the non-zero tract changes were positive. This suggests that, over our observed time range, more than half of brain region connections are developing relatively slowly and that a smaller subset of region-pairs are developing connections at a greater rate.

Further, we saw higher than average numbers for developing connections in the occipital and frontal lobes. Early development of neurons and connecting axonal fibers in the occipital lobe, prior to those in the frontal lobe, has been reported in the micro-scale brain anatomy literature [26, 51]. At the same time, Pandit et al. observed more connections developing with age between frontal lobe structures within 47 to 134 weeks post-conception [41]. It is possible that within the age range of this study, we are seeing both effects: the greater development in the occipital lobe could be occurring earlier in our cohort's age range, with greater development in the frontal lobe 
occurring later on. This hypothesis would confirm results reported by Takahashi et al., who found in postmortem infants, between 17 and 40 weeks post-conception, that emergence of connectivity moved generally from posterodorsal to anteroventral [50]. Unfortunately, the linear modeling we performed did not allow us to see these distinct periods of development separately. Our future work will look at exploring this hypothesis even further, either by fitting a higher order model or by examining smaller age ranges individually.

It is likely that the relative increases in tract count seen between certain region-pairs is primarily due oligodendrocyte maturation [16]. Myelin sheath maturation begins as early as 30 weeks but does not occur simultaneously throughout the brain, instead propagating up the corticospinal tracts and outwards, affecting major fiber bundles first [33]. We therefore expect then, in our cohort, that the majority of increased connectivity is due to oligodendrocyte maturation and not myelination.

We examined both tract-count connectomes and meanFA connectomes and found that there was significant overlap between developing edges between the two (section 3.2). This results is expected since higher FA suggests the presence of more cell structure connecting two regions [7]. However, high mean FA does not always imply higher oligodendrocyte maturation or that more neuronal fibers are connecting two regions. Regions of crossing fibers may have high fiber density but will present a low FA value in a standard DTI. That said, we expect that the effects of crossing fibers are less likely to present in this study given the early ages of the infants.

A notable difference between tract-count and mean-FA connectomes is that the mean-FA edge weights are not sensitive to relative bundle thickness. While certain fiber tracts may be thicker than others, they don't have to exhibit higher mean FA. For this reason, and those mentioned above, we conclude that there is value in examining both types of connectomes, especially when examining early infant development when a variety of complex processes may be influencing FA values.

The rapid development of cortico-cortical tracts found in our analysis of the normalized tract-count connectomes matches what is known in the literature. Long-range fibers between cortical regions develop across the corpus callosum between 33-35 weeks PCA and short-range corticocortical fibers begin developing after that [30]. Again, due to our linear model, here we only observe the combined effect of these two stages of development.

Examining the distance between regions of developing connections, we found that the majority of region-pairs exhibiting significantly increasing tract-counts were between spatially local regions $(<30 \mathrm{~mm})$. However, we did find some long-range connections between regions greater than $70 \mathrm{~mm}$ apart. This finding of a small number of long-range connections developing in the preterm infant brain is supported by the results of Takahashi et al., who also found such long range connections developing between 17 and 40 weeks gestation [50].

In section 3.3, it was shown that for tract-count connectomes, SW consistently increases with age across our age range. This result is consistent with results from Tymofiyeva et al. who found higher small-worldness in infants scanned shortly after normal term birth than in a group of preterm infants scanned at an average of $\sim 35$ weeks after conception [52]. In their work, as here, rise in smallworldness was due to increased normalized $\mathrm{CC}$ values and stable normalized CPL. van den Heuvel et al. also examined small-worldness in structural connectomes of preterm infants, scanned at 30 and 40 weeks PMA. Despite evidence of WMI and other pathologies in their cohort, our small-worldness results strongly agree with their findings of mean SW values of $\sim 1.3$ at 30 weeks and $\sim 1.5$ at 40 weeks [54]. While other studies have shown evidence of small-world brain networks at different ages, the fine temporal sampling of the dataset in our study exposed the clear trend of increasing SW and allowed detection of a significant positive correlation of SW with age [4]. This result suggests that the human connectome is less smallworld at an early stage in development, and perhaps at a very young age, not small-world at all.

In contrast, it was found that for mean-FA connectomes, SW was greater than 1 but did not increase with PMA. While many of the region-pair connections which exhibited a consistent increase in tract count also exhibited an increase in mean FA, there were a number of connections which exhibited an increase in FA but not in tract count (section 3.2). Thus, the mean-FA connectomes seemed to have extra connections, not present in the tract-count connectomes, that gained edge weights over time. It is likely that these extra connections with increasing edge weights are responsible for preventing increasing SW by inhibiting network segregation since the two connectome types seem to otherwise change similarly with age.

We also saw that not every subject's tract-count connectome increased in SW across age (section 3.4). This implies that either there is some error in our pipeline introducing a certain inaccuracy in connection weights, or that individual subjects develop somewhat distinctly and that the trend in increased small-worldness with age is not true for every person across every time-increment of early development. There is increased potential for this second option, given that the subjects studied here are all born preterm and, while were screened to be healthy, are likely affected in some way by the early age of birth. Nevertheless, it is impossible not to introduce some error in the registration, segmentation and tractography processes and thus it is probably the case that both explanations of decreased SW are, in part, responsible. Moving forward, there are certainly aspects of the connectome pipeline which can and should be improved, particularly through the use of more advanced tractography $[12,13]$ and registration techniques [11].

One subject in particular exhibited greatly decreased CC $(<-0.5)$ and was an outlier in this respect. This sub- 
ject also exhibited a relatively large increase in total tract count given the time between scans (i.e., it is well above the linear regression on the delta MWD plot in Figure 6). This large increase in number of tracts enables a large change in network topology between scans. However, the reason why, in this subject in particular, these new tracts are distributed across the network in a way that is causing reduced clustering is not clear since no other aspects of this subject, for which we have data, are notable.

One area not explored in this work is the effect of the birth age on the brain network properties examined here. While the cohort in this study was screened thoroughly to be normative, we still expect known effects of preterm birth, like reduced overall FA and increased MD, to have some effect on brain network connectivity [1, 3, 24]. A future direction is to examine the relationships between birth age, age at time of scan and connectome topology.

Also, it has been noted in the literature that when performing inference on multiple edges in a graph, like we did in Section 3.2, the topology of the graph can be used to more appropriately control the family-wise error rate (FWER) [59]. Network based statistics (NBS) by Zalesky et al. is a standard approach for leveraging network structure when controlling FWER but assumes a generalized linear model at each edge. Unfortunately, linear mixedeffects models are not within the class of generalized linear models. Future work may include extending NBS to linear mixed-effects models in order to extract more accurate inferences from datasets where each subject is scanned multiple times.

\section{Conclusions}

In this work, we analyzed the topology and longitudinal change in the structural connectomes of a cohort of young normative preterm neonates. To do this we constructed a pipeline to count the number of white matter tracts, and compute the mean FA, between pairs of anatomically defined regions in each scan. Individual connections and the high-level topology of the resulting brain networks were analyzed as a group and individually across age. Certain region-pair connections showed a particularly high rate of tract growth compared to others, particularly in the frontal and occipital lobes. However, discrepancies between tract-count and mean-FA connectome longitudinal trends exposed the importance of examining both measures of structural brain connectivity. Finally, we found that the preterm group in this study consistently exhibited high network integration and segregation and, most interestingly, significantly increasing small-worldness across age.

\section{Acknowledgements}

We thank the editors and reviewers of NeuroImage for their valuable and constructive feedback. We also thank
Dr. Ruth Grunau and Dr. Anne Synnes, as well as the staff of the Neonatal Follow-Up Program of BC Children's \& Women's Hospitals for their valuable contribution in assessing these children to confirm their neurodevelopmental outcomes.

CJB and GH were partially supported by the Natural Sciences and Engineering Research Council of Canada (NSERC) and BGB by Imperial Order Daughters of the Empire and Children of the Junior Branch (IODE) Canada and the Government of Alberta. SPM was supported by Canadian Institutes of Health Research (CIHR) operating grant MOP-79262. SPM is currently the Bloorview Children's Hospital Chair in Pediatric Neuroscience and was supported by a Tier 2 Canada Research Chair in Neonatal Neuroscience, and Michael Smith Foundation for Health Research Scholar.

\section{References}

[1] Mustafa Anjari, Latha Srinivasan, Joanna M. Allsop, Joseph V. Hajnal, Mary A. Rutherford, A. David Edwards, and Serena J. Counsell. Diffusion tensor imaging with tract-based spatial statistics reveals local white matter abnormalities in preterm infants. NeuroImage, 35:1021-1027, 2007.

[2] Gareth Ball, Paul Aljabar, Sally Zebari, Nora Tusor, Tomoki Arichi, Nazakat Merchant, Emma C Robinson, Enitan Ogundipe, Daniel Rueckert, A David Edwards, et al. Richclub organization of the newborn human brain. Proceedings of the National Academy of Sciences, 111(20):7456-7461, 2014.

[3] Gareth Ball, James P. Boardman, Paul Aljabar, Anand Pandit, Tomoki Arichi, Nazakat Merchant, Daniel Rueckert, A. David Edwards, and Serena J. Counsell. The influence of preterm birth on the developing thalamocortical connectome. Cortex, 49(6):1711 - 1721, 2013.

[4] Danielle Smith Bassett and ED Bullmore. Small-world brain networks. The neuroscientist, 12(6):512-523, 2006.

[5] Matteo Bastiani, Nadim Jon Shah, Rainer Goebel, and Alard Roebroeck. Human cortical connectome reconstruction from diffusion weighted mri: the effect of tractography algorithm. Neuroimage, 62(3):1732-1749, 2012.

[6] N. Bayley. Manual for the Bayley Scales of Infant Development. Harcourt, San Antonio, 3rd edition, 2006.

[7] Christian Beaulieu. The basis of anisotropic water diffusion in the nervous system - a technical review. NMR in Biomedicine, 15:435-455, 2002.

[8] Yoav Benjamini and Daniel Yekutieli. False discovery rateadjusted multiple confidence intervals for selected parameters. Journal of the American Statistical Association, 100(469):7181, 2005.

[9] Richard F Betzel, Alessandra Griffa, Andrea AvenaKoenigsberger, Joaquin Goni, Patric Hagmann, Jean-Phillippe Thiran, and Olaf Sporns. Multi-scale community organization of the human structural connectome and its relationship with resting-state functional connectivity. arXiv preprint arXiv:1304.0485, 2013.

[10] Sonia L Bonifacio, Hannah C Glass, Vann Chau, Jeffrey I Berman, Duan Xu, Rollin Brant, A James Barkovich, Kenneth J Poskitt, Steven P Miller, and Donna M Ferriero. Extreme premature birth is not associated with impaired development of brain microstructure. The Journal of pediatrics, 157(5):726$732,2010$.

[11] Brian G. Booth and Ghassan Hamarneh. Consistent information content estimation for diffusion tensor MR images. In Proceedings of Healthcare Informatics, Imaging and Systems Biology (HISB), pages 166-173, 2011.

[12] Brian G. Booth and Ghassan Hamarneh. Multi-region competitive tractography via graph-based random walks. In Proceedings 
of Mathematical Methods in Biomedical Image Analysis (MM$B I A)$, pages 73-78, 2012.

[13] Colin Brown, Brian G. Booth, and Ghassan Hamarneh. Kconfidence: Assessing uncertainty in tractography using k optimal paths. In IEEE International Symposium on Biomedical Imaging (IEEE ISBI), pages 250-253, 2013.

[14] Lin-Ching Chang, Derek K. Jones, and Carlo Pierpaoli. RESTORE: Robust estimation of tensors by outlier rejection. Magnetic Resonance in Medicine, 53:1088-1095, 2005.

[15] Vann Chau, Anne Synnes, Ruth E Grunau, Kenneth J Poskitt, Rollin Brant, and Steven P Miller. Abnormal brain maturation in preterm neonates associated with adverse developmental outcomes. Neurology, 81(24):2082-2089, 2013.

[16] Alexander Drobyshevsky, Sheng-Kwei Song, Georgi Gamkrelidze, Alice M Wyrwicz, Matthew Derrick, Fan Meng, Limin Li, Xinhai Ji, Barbara Trommer, Douglas J Beardsley, et al. Developmental changes in diffusion anisotropy coincide with immature oligodendrocyte progression and maturation of compound action potential. The Journal of neuroscience, 25(25):59885997, 2005.

[17] Jeroen Dudink, Jenny L. Kerr, Kathryn Paterson, and Serena J. Counsell. Connecting the developing preterm brain. Early $\mathrm{Hu}$ man Development, 84(12):777 - 782, 2008. Selected Proceedings of the Neonatal Update 2008 The Science of Newborn Care.

[18] M. Rhonda Folio and Rebecca R. Fewell. Peabody Developmental Motor Scales. PRO-Ed, 2nd edition, 2000.

[19] Peter Fransson, Ulrika Åden, Mats Blennow, and Hugo Lagercrantz. The functional architecture of the infant brain as revealed by resting-state fmri. Cerebral Cortex, 21(1):145-154, 2011.

[20] Peter Fransson, Beatrice Skiöld, Sandra Horsch, Anders Nordell, Mats Blennow, Hugo Lagercrantz, and Ulrika Åden. Resting-state networks in the infant brain. Proceedings of the National Academy of Sciences, 104(39):15531-15536, 2007.

[21] W Gao, W Lin, Y Chen, G Gerig, JK Smith, V Jewells, and JH Gilmore. Temporal and spatial development of axonal maturation and myelination of white matter in the developing brain. American journal of neuroradiology, 30(2):290-296, 2009.

[22] Wei Gao, Hongtu Zhu, Kelly S Giovanello, J Keith Smith, Dinggang Shen, John H Gilmore, and Weili Lin. Evidence on the emergence of the brain's default network from 2-week-old to 2year-old healthy pediatric subjects. Proceedings of the National Academy of Sciences, 106(16):6790-6795, 2009.

[23] Gaolang Gong, Yong He, Luis Concha, Catherine Lebel, Donald W Gross, Alan C Evans, and Christian Beaulieu. Mapping anatomical connectivity patterns of human cerebral cortex using in vivo diffusion tensor imaging tractography. Cerebral Cortex, 19(3):524-536, 2009.

[24] Petra S Hüppi, Stephan E Maier, Sharon Peled, Gary P Zientara, Patrick D Barnes, Ferenc A Jolesz, and Joseph J Volpe. Microstructural development of human newborn cerebral white matter assessed in vivo by diffusion tensor magnetic resonance imaging. Pediatric research, 44(4):584-590, 1998.

[25] Petra S Hüppi, Stephan E Maier, Sharon Peled, Gary P Zientara, Patrick D Barnes, Ferenc A Jolesz, and Joseph J Volpe. Microstructural development of human newborn cerebral white matter assessed in vivo by diffusion tensor magnetic resonance imaging. Pediatric Research, 44:584-590, 1998.

[26] Peter R Huttenlocher and Arun S Dabholkar. Regional differences in synaptogenesis in human cerebral cortex. Journal of comparative Neurology, 387(2):167-178, 1997.

[27] Mark Jenkinson, Peter Bannister, Michael Brady, and Stephen Smith. Improved optimization for the robust and accurate linear registration and motion correction of brain images. Neuroimage, $17(2): 825-841,2002$.

[28] Shuzhou Jiang, Hui Xue, Serena J Counsell, Mustafa Anjari, Joanna Allsop, Mary A Rutherford, Daniel Rueckert, and Joseph V Hajnal. In-utero three dimension high resolution fetal brain diffusion tensor imaging. In Medical Image Computing and Computer-Assisted Intervention-MICCAI 2007, pages 1826. Springer, 2007.
[29] Derek K. Jones and Peter J. Basser. "squashing peanuts and smashing pumpkins": How noise distorts diffusion-weighted MR data. Magnetic Resonance in Medicine, 52:979-993, 2004.

[30] Ivica Kostović and Nataša Jovanov-Milošević. The development of cerebral connections during the first 20-45 weeks gestation. In Seminars in Fetal and Neonatal Medicine, volume 11, pages 415-422. Elsevier, 2006.

[31] Vito Latora and Massimo Marchiori. Efficient behavior of smallworld networks. Physical review letters, 87(19):198701, 2001.

[32] Chun-Yi Lo, Pei-Ning Wang, Kun-Hsien Chou, Jinhui Wang, Yong He, and Ching-Po Lin. Diffusion tensor tractography reveals abnormal topological organization in structural cortical networks in alzheimer's disease. The Journal of Neuroscience, 30(50):16876-16885, 2010.

[33] Andrew Melbourne, Zach Eaton-Rosen, Alan Bainbridge, Giles S Kendall, Manuel Jorge Cardoso, Nicola J Robertson, Neil Marlow, and Sebastien Ourselin. Measurement of myelin in the preterm brain: Multi-compartment diffusion imaging and multi-component t2 relaxometry. In Medical Image Computing and Computer-Assisted Intervention-MICCAI 2013, pages 336-344. Springer, 2013.

[34] Leonard C Piecuch R Glidden DV Partridge JC et al. Miller SP, Ferriero DM. Early brain injury in premature newborns detected with magnetic resonance imaging is associated with adverse early neurodevelopmental outcome. Pediatrics, 147:609$616,2005$.

[35] Jeffrey J Neil, Shelly I Shiran, Robert C McKinstry, Georgia L Schefft, Avi Z Snyder, C Robert Almli, Erbil Akbudak, Joseph A Aronovitz, J Phillip Miller, BC Lee, et al. Normal brain in human newborns: apparent diffusion coefficient and diffusion anisotropy measured by using diffusion tensor $\mathrm{mr}$ imaging. Radiology, 209(1):57-66, 1998.

[36] Mark EJ Newman. Fast algorithm for detecting community structure in networks. Physical review E, 69(6):066133, 2004.

[37] Mark EJ Newman. Modularity and community structure in networks. Proceedings of the National Academy of Sciences, 103(23):8577-8582, 2006.

[38] Ann L Oberg and Douglas W Mahoney. Linear mixed effects models. In Topics in biostatistics, pages 213-234. Springer, 2007.

[39] Jukka-Pekka Onnela, Jari Saramäki, János Kertész, and Kimmo Kaski. Intensity and coherence of motifs in weighted complex networks. Physical Review E, 71(6):065103, 2005.

[40] Julia P Owen, Etay Ziv, Polina Bukshpun, Nicholas Pojman, Mari Wakahiro, Jeffrey I Berman, Timothy PL Roberts, Eric J Friedman, Elliott H Sherr, and Pratik Mukherjee. Test-retest reliability of computational network measurements derived from the structural connectome of the human brain. Brain connectivity, 3(2):160-176, 2013.

[41] AS Pandit, E Robinson, P Aljabar, G Ball, IS Gousias, Z Wang, JV Hajnal, D Rueckert, SJ Counsell, G Montana, et al. Wholebrain mapping of structural connectivity in infants reveals altered connection strength associated with growth and preterm birth. Cerebral Cortex, 2013.

[42] Burstein R Koffler H Papile LA, Burstein J. Incidence and evolution of subependymal and intraventricular hemorrhage: a study of infants with birth weights less than 1,500 gm. Pediatrics, 92:529-534, 1978.

[43] Josien PW Pluim, JB Antoine Maintz, and Max A Viergever. Mutual-information-based registration of medical images: a survey. Medical Imaging, IEEE Transactions on, 22(8):986-1004, 2003.

[44] Mikail Rubinov and Olaf Sporns. Complex network measures of brain connectivity: Uses and interpretations. NeuroImage, 52(3):1059 - 1069, 2010. Computational Models of the Brain.

[45] Ahmed Serag, Paul Aljabar, Gareth Ball, Serena J. Counsell, James P. Boardman, Mary A. Rutherford, A. David Edwards, Joseph V. Hajnal, and Daniel Rueckert. Construction of a consistent high-definition spatio-temporal atlas of the developing brain using adaptive kernel regression. NeuroImage, 59(3):2255 $-2265,2012$. 
[46] Feng Shi, Pew-Thian Yap, Guorong Wu, Hongjun Jia, John H Gilmore, Weili Lin, and Dinggang Shen. Infant brain atlases from neonates to 1-and 2-year-olds. PLoS One, 6(4):e18746, 2011.

[47] Ni Shu, Yaou Liu, Kuncheng Li, Yunyun Duan, Jun Wang, Chunshui Yu, Huiqing Dong, Jing Ye, and Yong He. Diffusion tensor tractography reveals disrupted topological efficiency in white matter structural networks in multiple sclerosis. Cerebral cortex, 21(11):2565-2577, 2011.

[48] Stephen M Smith. Fast robust automated brain extraction. Human brain mapping, 17(3):143-155, 2002.

[49] Kaustubh Supekar, Lucina Q. Uddin, Katherine Prater, Hitha Amin, Michael D. Greicius, and Vinod Menon. Development of functional and structural connectivity within the default mode network in young children. NeuroImage, 52(1):290 - 301, 2010.

[50] Emi Takahashi, Rebecca D Folkerth, Albert M Galaburda, and Patricia E Grant. Emerging cerebral connectivity in the human fetal brain: an mr tractography study. Cerebral Cortex, 22(2):455-464, 2012.

[51] Kate Teffer, Daniel P Buxhoeveden, Cheryl D Stimpson, Archibald J Fobbs, Steven J Schapiro, Wallace B Baze, Mark J McArthur, William D Hopkins, Patrick R Hof, Chet C Sherwood, et al. Developmental changes in the spatial organization of neurons in the neocortex of humans and common chimpanzees. Journal of Comparative Neurology, 521(18):42494259, 2013.

[52] Olga Tymofiyeva, Christopher P Hess, Etay Ziv, Patricia N Lee, Hannah C Glass, Donna M Ferriero, A James Barkovich, and Duan Xu. A dti-based template-free cortical connectome study of brain maturation. PloS one, 8(5):e63310, 2013.

[53] Olga Tymofiyeva, Christopher P Hess, Etay Ziv, Nan Tian, Sonia L Bonifacio, Patrick S McQuillen, Donna M Ferriero, A James Barkovich, and Duan Xu. Towards the baby connectome: mapping the structural connectivity of the newborn brain. PloS one, 7(2):e31029, 2012.

[54] Martijn P. van den Heuvel, Karina J. Kersbergen, Marcel A. de Reus, Kristin Keunen, Ren S. Kahn, Floris Groenendaal, Linda S. de Vries, and Manon J.N.L. Benders. The neonatal connectome during preterm brain development. Cerebral Cortex, 2014.

[55] R Wang, T Benner, AG Sorensen, and VJ Wedeen. Diffusion toolkit: a software package for diffusion imaging data processing and tractography. In Proc Intl Soc Mag Reson Med, volume 15, page 3720, 2007.

[56] Ze Wang, María Fernández-Seara, David C Alsop, Wen-Ching Liu, Judy F Flax, April A Benasich, and John A Detre. Assessment of functional development in normal infant brain using arterial spin labeled perfusion mri. Neuroimage, 39(3):973-978, 2008.

[57] Duncan J Watts and Steven H Strogatz. Collective dynamics of small-world networks. nature, 393(6684):440-442, 1998.

[58] Pew-Thian Yap, Yong Fan, Yasheng Chen, John H Gilmore, Weili Lin, and Dinggang Shen. Development trends of white matter connectivity in the first years of life. PLoS One, 6(9):e24678, 2011.

[59] Andrew Zalesky, Alex Fornito, and Edward $\mathrm{T}$ Bullmore. Network-based statistic: identifying differences in brain networks. Neuroimage, 53(4):1197-1207, 2010.

\section{Appendix A. Segmented regions and labels}

Table A1 lists the region names and abbreviations for the segmented regions from the UNC atlas, used in our connectome analysis. In Figure A.1, slices of the atlas with labeled regions are displayed visually for spatial context. 


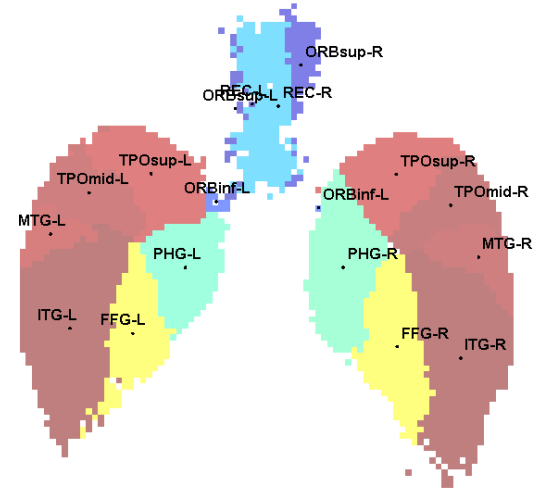

Slice 20

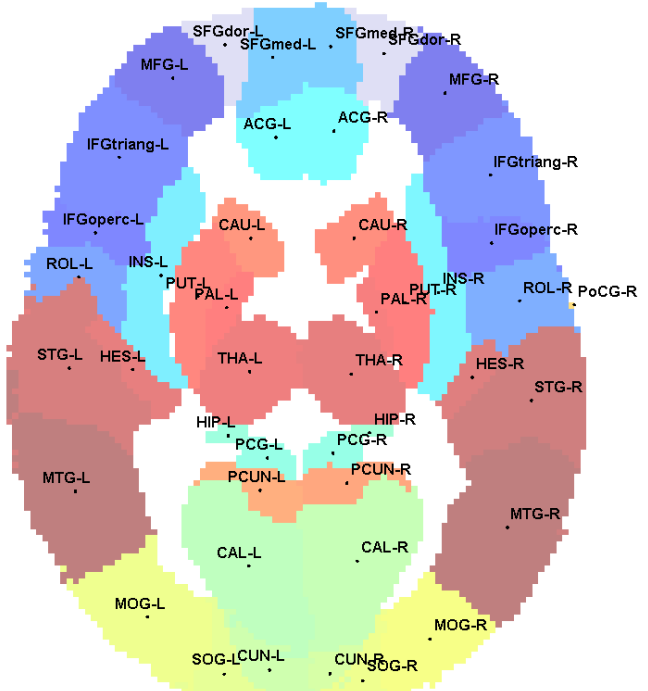

Slice 45

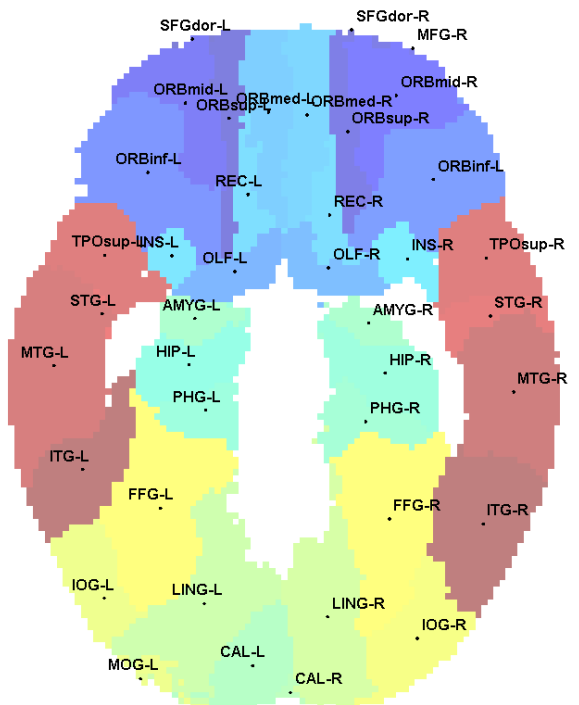

Slice 30

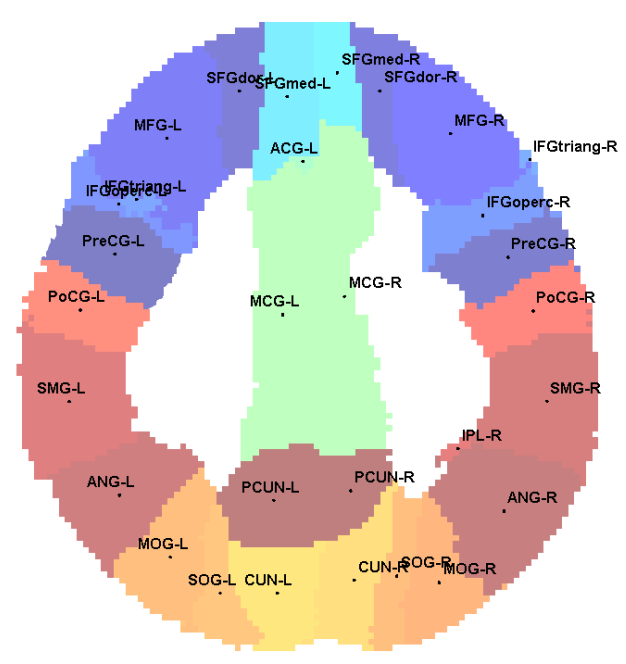

Slice 60

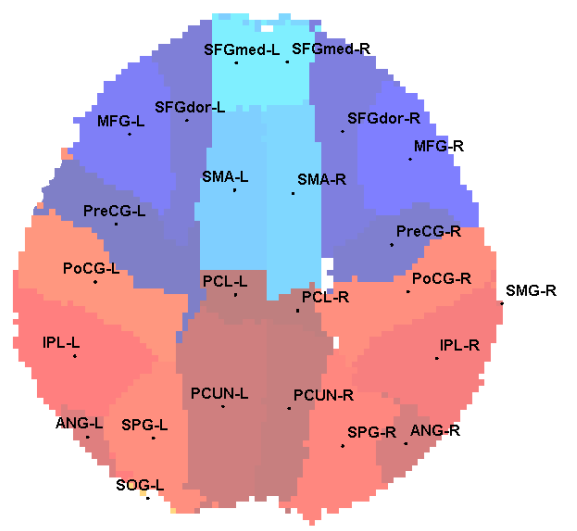

Slice 75

Figure A.1: Axial slices of the UNC atlas, numbered from inferior to superior. Each atlas region is coloured uniquely and labelled using a label from Table A1. 
Table A1: List of anatomical region names and abbreviations in UNC brain region atlas.

\begin{tabular}{|c|c|c|c|c|c|}
\hline Index & Region & Abrv. & Index & Region & Abrv. \\
\hline 1 & Precentral gyrus left & PreCG-L & 46 & Cuneus right & CUN-R \\
\hline 2 & Precentral gyrus right & PreCG-R & 47 & Lingual gyrus left & LING-L \\
\hline 3 & Superior frontal gyrus (dorsal) left & SFGdor-L & 48 & Lingual gyrus right & LING-R \\
\hline 4 & Superior frontal gyrus (dorsal) right & SFGdor-R & 49 & Superior occipital gyrus left & SOG-L \\
\hline 5 & Orbitofrontal cortex (superior) left & ORBsup-L & 50 & Superior occipital gyrus right & SOG-R \\
\hline 6 & Orbitofrontal cortex (superior) right & ORBsup-R & 51 & Middle occipital gyrus left & MOG-L \\
\hline 7 & Middle frontal gyrus left & MFG-L & 52 & Middle occipital gyrus right & MOG-R \\
\hline 8 & Middle frontal gyrus right & MFG-R & 53 & Inferior occipital gyrus left & IOG-L \\
\hline 9 & Orbitofrontal cortex (middle) left & ORBmid-L & 54 & Inferior occipital gyrus right & IOG-R \\
\hline 10 & Orbitofrontal cortex (middle) right & ORBmid-R & 55 & Fusiform gyrus left & FFG-L \\
\hline 11 & Inferior frontal gyrus (opercular) left & IFGoperc-L & 56 & Fusiform gyrus right & FFG-R \\
\hline 12 & Inferior frontal gyrus (opercular) right & IFGoperc-R & 57 & Postcentral gyrus left & PoCG-L \\
\hline 13 & Inferior frontal gyrus (triangular) left & IFGtriang-L & 58 & Postcentral gyrus right & PoCG-R \\
\hline 14 & Inferior frontal gyrus (triangular) right & IFGtriang-R & 59 & Superior parietal gyrus left & SPG-L \\
\hline 15 & Orbitofrontal cortex (inferior) left & ORBinf-L & 60 & Superior parietal gyrus right & SPG-R \\
\hline 16 & Orbitofrontal cortex (inferior) right & ORBinf-L & 61 & Inferior parietal lobule left & IPL-L \\
\hline 17 & Rolandic operculum left & ROL-L & 62 & Inferior parietal lobule right & IPL-R \\
\hline 18 & Rolandic operculum right & ROL-R & 63 & Supramarginal gyrus left & SMG-L \\
\hline 19 & Supplementary motor area left & SMA-L & 64 & Supramarginal gyrus right & SMG-R \\
\hline 20 & Supplementary motor area right & SMA-R & 65 & Angular gyrus left & ANG-L \\
\hline 21 & Olfactory left & OLF-L & 66 & Angular gyrus right & ANG-R \\
\hline 22 & Olfactory right & OLF-R & 67 & Precuneus left & PCUN-L \\
\hline 23 & Superior frontal gyrus (medial) left & SFGmed-L & 68 & Precunesu right & PCUN-R \\
\hline 24 & Superior frontal gyrus (medial) right & SFGmed-R & 69 & Precentral lobule left & PCL-L \\
\hline 25 & Orbitofrontal cortex (medial) left & ORBmed-L & 70 & Precentral lobule right & PCL-R \\
\hline 26 & Orbitofrontal cortex (medial) right & ORBmed-R & 71 & Caudate left & CAU-L \\
\hline 27 & Rectus gyrus left & REC-L & 72 & Caudate right & CAU-R \\
\hline 28 & Recuts gyrus right & REC-R & 73 & Putamen left & PUT-L \\
\hline 29 & Insula left & INS-L & 74 & Putamen right & PUT-R \\
\hline 30 & Insula right & INS-R & 75 & Pallidum left & PAL-L \\
\hline 31 & Anterior cingulate gyrus left & ACG-L & 76 & Pallidum right & PAL-R \\
\hline 32 & Anterior cingulate gyrus right & ACG-R & 77 & Thalamus left & THA-L \\
\hline 33 & Middle cingulate gyrus left & MCG-L & 78 & Thalamus right & THA-R \\
\hline 34 & Middle cingulate gyrus right & MCG-R & 79 & Heschl gyrus left & HES-L \\
\hline 35 & Posterior cingulate gyrus left & PCG-L & 80 & Heschl gyrus right & HES-R \\
\hline 36 & Posterior cingulate gyrus right & PCG-R & 81 & Superior temporal gyrus left & STG-L \\
\hline 37 & Hippocampus left & HIP-L & 82 & Superior temporal gyrus right & STG-R \\
\hline 38 & Hippocampus right & HIP-R & 83 & Temporal pole (superior) left & TPOsup-L \\
\hline 39 & ParaHippocampal gyrus left & PHG-L & 84 & Temporal pole (superior) right & TPOsup-R \\
\hline 40 & ParaHippocampal gyrus right & PHG-R & 85 & Middle temporal gyrus left & MTG-L \\
\hline 41 & Amygdala left & AMYG-L & 86 & Middle temporal gyrus right & MTG-R \\
\hline 42 & Amygdala right & AMYG-R & 87 & Temporal pole (middle) left & TPOmid-L \\
\hline 43 & Calcarine cortex left & CAL-L & 88 & Temporal pole (middle) right & TPOmid-R \\
\hline 44 & Calcarine cortex right & CAL-R & 89 & Inferior temporal gyrus left & ITG-L \\
\hline 45 & Cuneus left & CUN-L & 90 & Inferior temporal gyrus right & ITG-R \\
\hline
\end{tabular}

